\title{
Influence of Incision Site on Postoperative Outcome in Skin-/Nipple-Sparing Mastectomy: Is There a Difference between Radial and Inframammary Incision?
}

\author{
Monika Lanthaler ${ }^{a}$ Rossella Spinelli ${ }^{a} \quad$ Christoph Tasch ${ }^{a}$ Michael Sieb ${ }^{b}$ \\ Manuel Harfmann ${ }^{a} \quad$ Agnese Nitto $^{a}$ Gerhard Pierer $^{\mathrm{a}}$ Thomas Bauer ${ }^{\mathrm{a}}$

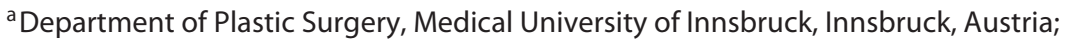 \\ ${ }^{b}$ Department of Mechatronics - Medical Engineering, Management Center Innsbruck, Innsbruck, Austria
}

\section{Keywords}

Breast reconstruction · Incision site $\cdot$ Aesthetic results

\begin{abstract}
Background: The aim of this study was to determine whether there is a difference in results between the radial and the inframammary approach in nipple-/skin-sparing mastectomy and immediate reconstruction. Methods: The patients were divided into two groups (group 1: radial incision; group 2: inframammary fold incision [IMF]), each consisting of two subgroups for direct-to-implant reconstruction $(1 \mathrm{a}, 2 \mathrm{a})$ and expander reconstruction (1b, 2b). The patients were operated on between March 2012 and May 2017. Preoperative tumor parameters, reconstruction parameters, postoperative tumor parameters, and immediate and late complications were assessed. Postoperative photographs were evaluated by the patients and 8 plastic surgeons by means of grading (1-5) and the visual analog scale (VAS; $1-10)$. $\boldsymbol{R e}$ sults: Enrolled in this study were 28 patients, namely, 7 patients in each subgroup. The median age was 46 years, and the median follow-up period was 40 months. No immediate complications occurred. Three patients had late complications, but there was no case of evident capsular fibrosis. Twenty-seven patients (96.42\%) evaluated the postoperative result as excellent/good. The postoperative evaluation by the plastic surgeons was excellent/good for a median of 18 patients (64.28\%). Group 1 showed a median VAS score of 7.63 (expander group: 7.50; direct-to-implant group: 7.75); the median VAS score for group 2 was 8.25 (expander group:
\end{abstract}

8.75; direct-to-implant group: 7.50). Conclusions: Our study shows good results for implant breast reconstruction in both groups, with minimally better results for the IMF group. In the direct-to-implant subgroups, the radial group showed slightly better results.

() 2019 S. Karger AG, Basel

\section{Introduction}

The benefits of immediate reconstruction include an improved aesthetic outcome, less psychological stress, and the advantage of simultaneous completion of mastectomy and reconstruction [1]. As skin-sparing mastectomy (SSME) and nipple-sparing mastectomy (NSME) have been proven to be oncologically safe, the number of immediate breast reconstructions $[2,3]$ has been increasing during recent years. As compared with autologous reconstruction, the relative simplicity, rapid recovery, and exclusion of donor-site morbidity for expander/implant breast reconstruction are often appealing [4].

One-stage direct-to-implant immediate breast reconstruction is recommended for small-to-medium nonptotic breasts with well-vascularized skin flaps, but contraindicated in patients treated with radiotherapy. Flap necrosis, reoperation, and implant loss are generally more common with direct-to-implant reconstruction $[5,6]$, but there is the advantage that no further operation is re-

M. Lanthaler and R. Spinelli contributed equally as first authors. 
quired and recovery is thus faster [7]. Nevertheless, some authors report that the likelihood of requiring a secondary procedure is not lower after one-stage implant reconstruction [8].

In order to achieve good breast reconstruction results, appropriate patient selection is mandatory. As described in the literature, poor results are more common with a higher BMI, smoking, and poorly controlled diabetes mellitus [9]. The ideal patient for NSME and immediate expander/implant reconstruction is relatively smallbreasted, with little or no ptosis, undergoing bilateral mastectomy $[10,11]$.

As, to our knowledge, no paper to date has investigated the influence of incision site on the final operative result and we felt this topic is relevant, we started this study with the aim of determining whether there is a difference in postoperative outcome between the radial and the inframammary fold approach (IMF) in NSME/SSME.

We hypothesized that the radial approach provides better aesthetic results, as we feel that it allows the shape of the breast to be reconstructed better because the radial approach permits the implant to be brought into a better inferior and medial position. Additionally, wound healing with the muscle underneath the skin seems to be better. An apparent disadvantage is the more visible scar in comparison to the inframammary approach.

Our primary aim was to have plastic surgeons evaluate actual postoperative photographs of patients whose reconstruction had been completed at least 1 year previously. In addition to grading the postoperative results, we opted to objectify the results through visual analog scale (VAS) score evaluation. In addition to the doctors' evaluation, we wanted the patients to also evaluate their postoperative aesthetic result by grading. Moreover, we assessed early (hematoma, skin necrosis, or infection) and late complications (capsular fibrosis, implant rupture, and aesthetic dissatisfaction).

\section{Subjects and Methods}

This study included 28 patients. The patients were divided into two groups (group 1: radial incision; group 2: IMF incision), each consisting of two subgroups: group la (radial group with primary implant [RGI]), group $1 \mathrm{~b}$ (radial group with primary expander [RGE]), group $2 \mathrm{a}$ (inframammary fold approach with primary implant [IMFI]), and group $2 \mathrm{~b}$ (inframammary fold approach with primary expander [IMFE]).

The study design is retrospective explorative. The patients included in this study were operated on between March 2012 and May 2017. We opted for a postoperative observation period of at least 1 year. We excluded patients with tumor recurrence for ethical reasons, and also excluded patients with simultaneous reduction or mastopexy through vertical or $\mathrm{T}$ incision. Our aim was to have a group that was as homogeneous as possible. For this reason, we initially planned to include only bilateral reconstruction cases. However, as we saw that it was very difficult to reach patients and motivate them to come for a renewed checkup, we had to add unilateral reconstruction cases to each subgroup in order to have 7 patients in each subgroup.

For each group, preoperative tumor parameters (preoperative chemotherapy and radiotherapy), general patient characteristics (age, BMI, diabetes mellitus, and smoking), reconstruction parameters (resection weight, implant volume, and TiLOOP ${ }^{\circledR}$ matrix used), and postoperative tumor parameters (postoperative radiotherapy and chemotherapy) were assessed. Immediate (bleeding, infection, and skin necrosis) and late complications (capsular fibrosis, implant displacement, and implant loss) were evaluated.

Included in this study were only patients who returned for routine follow-up at least 1 year after completion of breast reconstruction. Our primary aim was to compare aesthetic outcome by evaluating postoperative pictures. Overall aesthetic results, breast volume, breast symmetry, ptosis, location of the areola, and breast projection were evaluated by grading (1-5), with "excellent" for the best and "poor" for the worst grade. The grades given by all 8 plastic surgeons when evaluating each patient were added to form one median value. In addition to grading the postoperative result, we opted to objectify the assessment by means of VAS score evaluation (VAS scale, 1-10), with 1 for "worst" and 10 for "best."

In addition to the doctors' evaluation, we wanted the patients to also grade their postoperative aesthetic result, with 1 for "excellent" and 5 for "poor." Patients' satisfaction with the operative result was also related to resection weight, implant volume, whether chemotherapy had to be administered, and BMI.

\section{Surgical Methods}

All expanders/implants were placed in a submuscular pocket. Anatomically textured implants or expanders with integrated valves were used. Nonresorbable TiLOOP ${ }^{\circledR}$ meshes [12] were used to cover the silicon implant in the lower lateral quadrant. In the case of expander reconstruction, postoperative expansion began 14-21 days after surgery, and the switch from expander to permanent implant was performed after a minimum of 6 months postoperatively.

Statistical calculations were performed using SPSS version 24 for Windows.

\section{Results}

The median age of the 28 patients was 46 years. In the radial group, the median age was 52 years; in the IMF group, the patients were younger at a median age of 40 years. The median follow-up period from completion of reconstruction was 40 months (RGI: 33 months; RGE: 37 months; IMFI: 28 months; IMFE: 49 months).

Among the 28 patients there were 6 prophylactic bilateral mastectomies due to gene mutation and 13 prophylactic contralateral operations with tumor occurrence on the other side; 1 patient presented with a tumor on each side, and 8 patients underwent unilateral mastectomy for tumor resection.

The median resection weight of the specimens in group la (RGI) was $180 \mathrm{~g}$ with an implant volume of 180 $\mathrm{cm}^{3}$. In group $1 \mathrm{~b}$ (RGE), the resection weight was $300 \mathrm{~g}$ and the implant volume $320 \mathrm{~cm}^{3}$, showing that the implant volume was considerably larger in the expander 


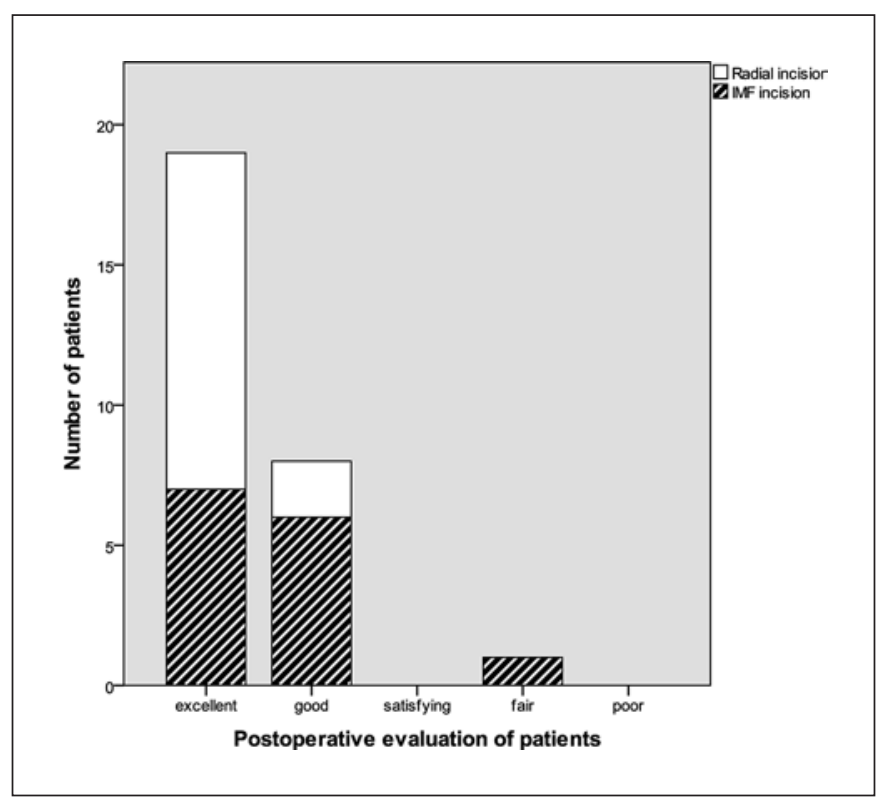

Fig. 1. Postoperative evaluation by the patients. IMF, inframammary fold.

group. The median resection weight in group $2 \mathrm{a}$ (IMFI) was $182 \mathrm{~g}$ with a median implant volume of $300 \mathrm{~cm}^{3}$. In the IMFE group (2b), the median resection weight was $260 \mathrm{~g}$ with a considerably larger final implant volume at a median of $425 \mathrm{~cm}^{3}$. Overall, the IMF group showed a larger implant volume than did the radial group, and in both groups the expander group had larger implants.

The median BMI in the radial group was $20 \mathrm{~kg} / \mathrm{m}^{2}$ in group $1 \mathrm{a}$ and $21.5 \mathrm{~kg} / \mathrm{m}^{2}$ in group $1 \mathrm{~b}$; in the IMF group, it was $22 \mathrm{~kg} / \mathrm{m}^{2}$ in group $2 \mathrm{a}$ and $21.3 \mathrm{~kg} / \mathrm{m}^{2}$ in group $2 \mathrm{~b}$. In all, most patients had a fairly normal weight. The expander groups consisted of slightly higher-weight patients than the direct-to-implant groups.

Eight of the 28 patients were active smokers, none of whom had wound healing problems. No diabetes mellitus was seen.

In the radial group, 4 patients had undergone neoadjuvant and/or adjuvant chemotherapy (group 1a: 3 patients; group 1b: 1 patient), whereas in the IMF group, 6 patients ( 3 in group $2 \mathrm{a}$ and 3 in group $2 \mathrm{~b}$ ) had to undergo chemotherapy. One patient with necrosis had undergone pre- and postoperative chemotherapy (group 1a).

\section{Postoperative Breast Symmetry}

None of the 8 unilaterally operated patients had contralateral breast symmetrization, and only 1 patient, who had severe ptosis of the contralateral breast, was unsatisfied with breast symmetry. Four of the 8 patients underwent SSME through a radial incision with primary expander reconstruction; 2 further radial approaches were performed with direct-to-implant reconstruction. All

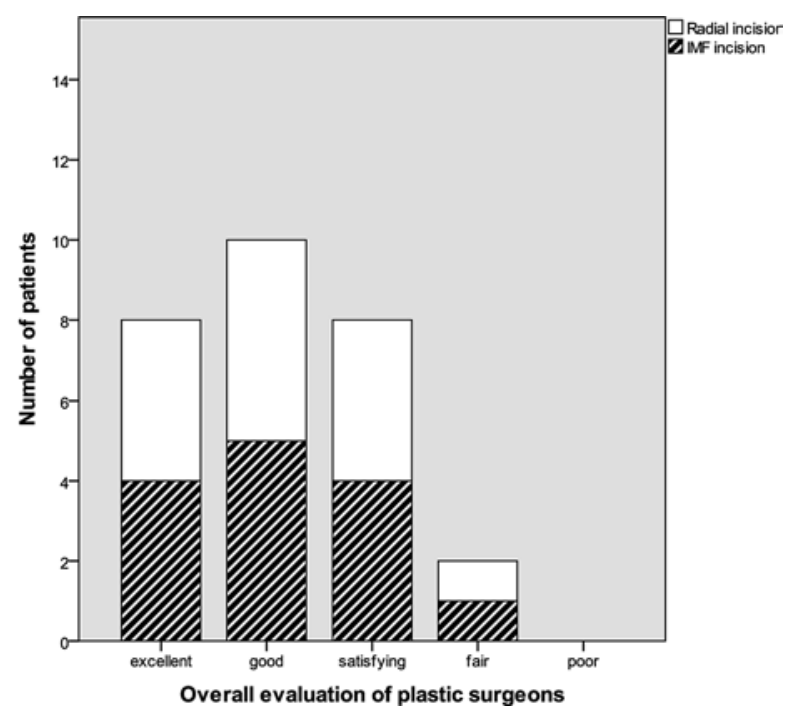

Fig. 2. Overall evaluation by the plastic surgeons. IMF, inframammary fold.

these patients showed good results concerning symmetry. The 2 remaining patients had been treated via an inframammary approach with direct-to-implant reconstruction, and one of them was unsatisfied with the severe ptosis of the other side. In light of these results, we have the impression that especially patients having undergone SSME tend to show good results concerning symmetry, also with unilateral reconstruction.

In the IMFI group, 1 patient underwent aesthetic augmentation before cancer was diagnosed and had NSME with immediate implant reconstruction thereafter with an excellent outcome.

\section{Postoperative Complications}

There were no immediate complications (bleeding, infection, or severe seroma). Only 2 patients in the radial group (1 patient each in group la and group $1 \mathrm{~b}$ ) had minor operative wound revisions.

Only 1 patient in the immediate implant group with a radial incision presented with skin necrosis. This was the only major reoperation necessary in either group. This patient was a nonsmoker but had undergone pre- and postoperative chemotherapy.

In our 28 nonradiated operated patients, no case of evident capsular fibrosis was observed.

\section{Postoperative Evaluation by the Patients}

When evaluating the postoperative results, 19 patients (67.85\%; 12 patients in the radial group: 6 in RGI and 6 in RGE; 7 patients in the IMF group: 3 in IMFI and 4 in IMFE) rated their postoperative results as "excellent." 


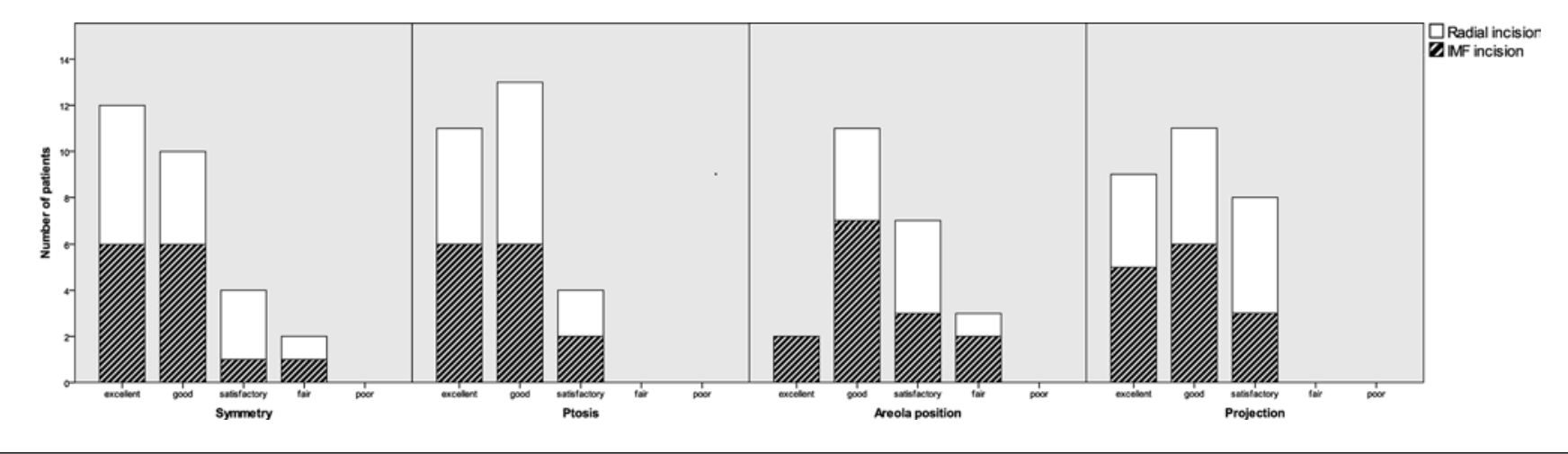

Fig. 3. Evaluation of symmetry, ptosis, areola position, and breast projection by the plastic surgeons. IMF, inframammary fold.

Table 1. Patient satisfaction regarding resection weight, implant volume, chemotherapy, and BMI

\begin{tabular}{|c|c|c|c|c|c|c|}
\hline & & \multicolumn{5}{|c|}{ Patient satisfaction } \\
\hline & & excellent & good & satisfactory & fair & poor \\
\hline \multirow[t]{2}{*}{ Resection weight } & $<300 \mathrm{~g}$ & 13 & 4 & 0 & 0 & 0 \\
\hline & $\geq 300 \mathrm{~g}$ & 6 & 4 & 0 & 1 & 0 \\
\hline \multirow[t]{2}{*}{ Implant volume } & $<300 \mathrm{~cm}^{3}$ & 10 & 3 & 0 & 0 & 0 \\
\hline & $\geq 300 \mathrm{~cm}^{3}$ & 9 & 5 & 0 & 1 & 0 \\
\hline \multirow[t]{2}{*}{ Preoperative chemotherapy } & yes & 6 & 3 & 0 & 0 & 0 \\
\hline & no & 13 & 5 & 0 & 1 & 0 \\
\hline \multirow[t]{2}{*}{ Postoperative chemotherapy } & yes & 4 & 3 & 0 & 0 & 0 \\
\hline & no & 15 & 5 & 0 & 1 & 0 \\
\hline \multirow[t]{2}{*}{$\mathrm{BMI}$} & $<25 \mathrm{~kg} / \mathrm{m}^{2}$ & 17 & 7 & 0 & 0 & 0 \\
\hline & $\geq 25 \mathrm{~kg} / \mathrm{m}^{2}$ & 2 & 1 & 0 & 1 & 0 \\
\hline
\end{tabular}

The result was classified as "good" by 8 further patients (28.57\%; 2 patients in the radial group: 1 in RGI and 1 in RGE; 6 patients in the IMF group: 3 in IMFI and 3 in IMFE). Only 1 patient (3.5\%) classified her result as "fair"; she was in the IMFI group. Overall, 27 (96.42\%) of the patients evaluated their postoperative result as "excellent" or "good". The postoperative evaluation by the patients is shown in Figure 1.

Only 2 patients assessed postoperative symmetry as unsatisfactory, whereas the remaining 26 patients were satisfied (14 in the radial group and 12 in the IMF group); the results were slightly better for the radial group. Patient satisfaction with the postoperative result was also evaluated with regard to resection weight, implant volume, administered chemotherapy, and BMI. Of the 19 patients who evaluated their postoperative result as "excellent," 13 had a resection weight $<300 \mathrm{~g}, 10 \mathrm{had}$ an implant volume $<300 \mathrm{~cm}^{3}$, only 7 had to undergo chemotherapy, and 17 had a BMI $<25 \mathrm{~kg} / \mathrm{m}^{2}$ (Table 1 ).

\section{Postoperative Evaluation by the Plastic Surgeons by Means of Grading}

When the postoperative photographs were evaluated by the experienced plastic surgeons, the 8 surgeons' evaluations for each patient were combined into one median value. The overall evaluation showed a median grading of "excellent" for 8 patients ( 4 in the radial group: 2 in RGI and 2 in RGE; 4 in the IMF group: 2 in IMFI and 2 in IMFE), "good" for 10 patients (5 in the radial group: 3 in RGI and 2 in RGE; 5 in the IMF group: 1 in IMFI and 4 in IMFE), "satisfactory" for 8 patients (4 in the radial group: 1 in RGI and 3 in RGE; 4 in the IMF group: 3 in IMFI and 1 in IMFE), "fair" for 1 patient in the radial group and 1 patient in the IMF group (both in the respective direct-to-implant subgroup), and "poor" for no patient (Fig. 2). Altogether, the median evaluation was "excellent" or "good" for 18 of the 28 patients. Among these 18 patients, there were 9 radial and 9 IMF approaches and altogether 10 expander reconstructions. The 8 plastic sur- 


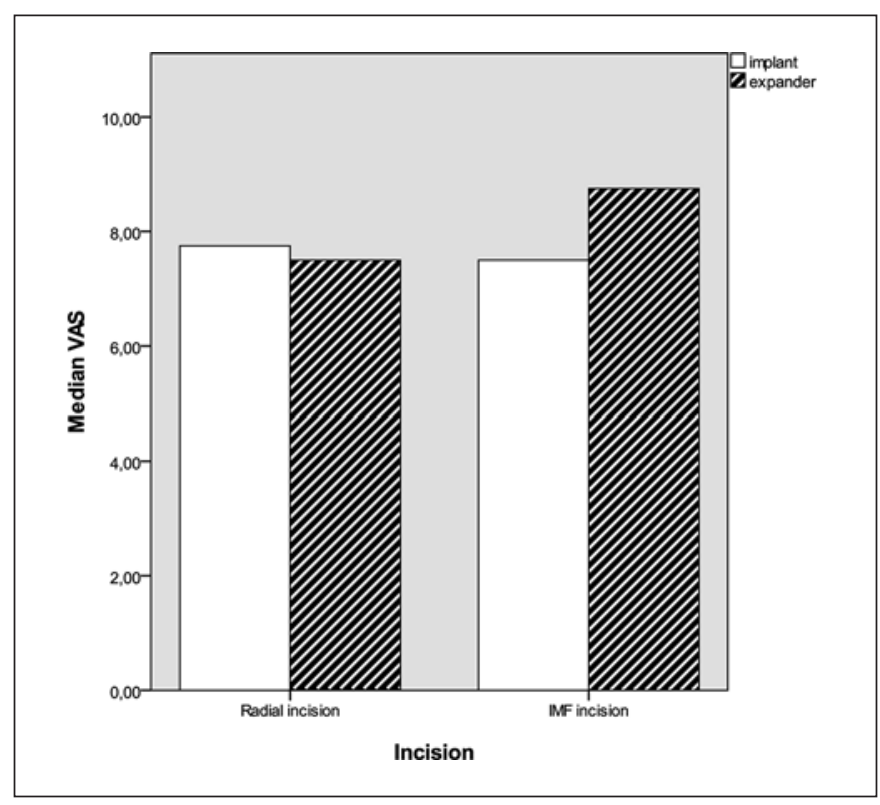

Fig. 4. VAS score evaluation by the plastic surgeons. IMF, inframammary fold; VAS, visual analog scale.

geons also evaluated breast volume, breast symmetry, ptosis, areola position, and breast projection by means of grading (Fig. 3).

When regarding the "excellent" results alone, the radial group and the IMF group showed the same results. The "excellent" and "good" ratings together (18 patients) also showed similar results, with 9 patients in each group consisting of 6 expander reconstructions in the IMF group and 4 expander reconstructions in the radial group, showing again that especially in the IMF group, expander reconstruction provided slightly better results.

Postoperative VAS Evaluation by the Plastic Surgeons

When regarding the median VAS scores given by the 8 plastic surgeons, the radial group (group 1) had a median VAS score of 7.63 (RGE: 7.50; RGI: 7.75), thus showing quite the same results for the expander and the directto-implant procedure, with slightly better results for RGI. The VAS evaluation of the IMF group (median score: 8.25) showed slightly better results than for the radial group. In the IMF group, IMFE (with a VAS score of 8.75) showed better results than IMFI (with a VAS score of 7.50) (Fig. 4).

\section{Discussion}

The reliability of expander/implant reconstruction has been demonstrated in many studies. Cordeiro and McCarthy [11] showed that $90 \%$ of the patients in his series had a good-to-excellent aesthetic result after a mean follow-up of 3 years. Bilateral reconstructions had a significantly higher overall aesthetic score. In a review of $52 \mathrm{ex}-$ pander/implant reconstructions, Ramon et al. [13] demonstrated that good-to-excellent results were achieved in $75 \%$ of their patients. Cavalcante and Lima [14] reported very satisfying aesthetic results with a $>90 \%$ good/excellent rating.

Some oncologists generally prefer radial lateral incision as it allows better access to the breast, nipple-areolar complex, and axilla. Contrarily, the inframammary approach yields a less evident scar hidden in the inframammary crease.

Although many surgeons perform NSME and immediate implant reconstruction in a single stage, many surgeons prefer two-stage expander-to-implant reconstruction, as it reduces complications and the outcome is more predictable $[10,14,15]$. The ideal patient for NSME and immediate expander/implant reconstruction is relatively small-breasted, with little or no ptosis, and undergoing bilateral risk-reducing mastectomy [16]. As described in the literature, an implant volume $>300 \mathrm{~cm}^{3}$, obesity, severe ptosis of the breast, older age, and smoking may lead to poor aesthetic results [17] and skin necrosis. Such patients may need more revisional operations after directto-implant reconstruction than they would have had with the two-stage operation performed initially [8].

Our study group of 28 patients assessed for the purpose of comparing the radial and the inframammary approach - the latter of which is hardly presented in the literature - was very small. When we started our study in May 2018, we attempted to include as many patients as possible and therefore looked up all patients who had been operated on for implant reconstruction between March 2012 and May 2017. We did not want to include patients operated on at a later date so as to have a postoperative follow-up of at least 1 year after completion of treatment, since we felt that with a shorter follow-up it would be difficult to draw any firm conclusions. For ethical reasons, we excluded all patients who had tumor recurrence. Moreover, we excluded patients with simultaneous reduction or mastopexy through vertical or $\mathrm{T}$ incision, as we feel this is a completely different group with frequent perfusion problems of the skin envelope or the areola and consecutive wound healing problems. By including only patients treated with radial incision and IMF incision, we intended to have a group as homogeneous as possible. At our hospital, we perform expander and direct-to-implant reconstruction, and we therefore wanted to include these subgroups because we felt it is important to distinguish them as subgroups in order to be able to draw any firm conclusions. There were many patients who did not come to their checkup, and when we contacted them, many were not willing to come for a renewed 
checkup. Many of them did not want to come to the hospital, since they associate a hospital visit with their breast cancer and they did not want to be reminded of it.

Another reason for the small number of patients certainly is the fact that at our institution breast reconstruction is a very individualized approach for every patient, using the whole armamentarium of the reconstructive surgeon, such as oncoplastic surgery, implants, expanders, fat grafting, and local and free flaps, adapting the procedure to the patient's needs and wishes. Therefore, it was not possible to find larger numbers of comparable individuals. However, as we view the topic of our study as relevant and because it is hardly presented in the literature, we conducted our study with as many patients we were able to enroll.

Our study group consisted of fairly normal-weighted individuals, and the group with the highest resection weight was RGE (median resection weight: $300 \mathrm{~g}$ ). The largest implant volume was seen in IMFE, with a median implant weight of $425 \mathrm{~cm}^{3}$, whereas in RGE it was smaller (median: $320 \mathrm{~cm}^{3}$ ). We suppose that use of a larger implant volume was an attempt to correct ptosis, and as our data reflect this, it seemed to be necessary more often in the IMF group. One reason may be that radial incision permits the skin envelope to be distributed better over the whole breast mound as compared with the IMF approach.

The complication rate in our study group was very low, with an early complication rate of only 3 operative revisions among 28 patients. Also the long-term complication results, with no case of capsular fibrosis, were excellent - although with regard to capsular fibrosis, a 1-year follow-up is too short to make a definite evaluation. We also have to remember that our study group included no patient undergoing radiation therapy, as at our department, we normally opt for deep inferior epigastric perforator or profunda artery perforator reconstruction if radiation is planned. Our results are also in accordance with a recently published study by Cavalcante and Lima [14], who after a low radiation rate of $6.4 \%$ describe no capsular contractures of grade III or IV in their study group when evaluating postoperative aesthetic results.

Contrary to our assumption that radial incision would show better aesthetic results with regard to the long-term VAS evaluation by our 8 plastic surgeons, the IMF group showed slightly better results than the radial group (VAS score: 8.25 vs. 7.63 ). However, when regarding the radial group alone, RGI showed slightly better results (VAS score: 7.75) than RGE (VAS score: 7.50). This may lead to the conclusion that, especially in IMF patients, if intraoperative conditions are not optimal, we should rather tend to use expander reconstruction. We suppose that with the inframammary approach and direct-to-implant reconstruction, the lower pole tends to tighten and the implant may tend to be placed too high. By contrast, with radial incision direct-to-implant reconstruction, the lower pole may extend better. Moreover, when switching from expander to implant in radial incision, it is very easy to lower the IMF, in contrast to IMF incision. It is therefore possible that direct-to-implant reconstruction in some difficult patients is tolerated better with the radial approach than with the inframammary approach, as the VAS scores for the subgroups show. However, in candidates well suited to direct-to-implant reconstruction, the IMF approach is a good option, as is reflected in our data showing a slightly better overall VAS score for the IMF group.

Overall, 20 patients (71.42\%) evaluated their postoperative result as "excellent" or "good," 14 of whom had undergone primary expander reconstruction. Most patients with good postoperative evaluations had no chemotherapy, a BMI $<25 \mathrm{~kg} / \mathrm{m}^{2}$, and a resection weight $<300 \mathrm{~g}$.

As seen from the symmetry evaluation by our patients, the radial incision leads to less pexy effect in ptotic breasts and thus seems to tolerate more ptosis on the contralateral side. Nevertheless, all in all, the IMF approach showed slightly better results in terms of VAS scores. Of the 20 patients assessed as having "excellent" or "good" results, 11 had undergone primary expander reconstruction, thus showing slightly better results for expander reconstruction.

When evaluating the photographs, we also had the impression that primary expander reconstruction permits an irregular skin envelope to be corrected better, the areola to be positioned more centrally, the IMF to be better defined, and a larger volume to be implanted with a minimal risk of skin envelope necrosis, as seen from the largest implants in IMFE (median: $425 \mathrm{~cm}^{3}$ ) in our youngest patients.

All in all, both the radial group and the IMF group showed quite good results, with most patients being satisfied. From our patients' evaluations, with 19 of them $(67.85 \%)$ evaluating their result as "excellent" and 8 (28.57\%) as "good," we conclude that implant/expander reconstruction is a good reconstructive option in breast surgery, with a large number of satisfied patients, a low complication rate, a short operative time, and quick recovery.

The results were nearly the same for both groups, with slightly better results for the IMF group. However, since we may see from the VAS evaluation in the radial group that there were slightly better results in RGI than in RGE, we may conclude that especially in the case of ptosis, radial incision sometimes offers better results with the direct-to-implant approach, as this approach permits us to also minimally lift the breast. This could be supported by lowering the IMF fold. Another possibility of correcting mild ptosis with the IMF approach would be to use larger 
implants - as, we feel, was automatically done in our IMF study group with the larger implant volume used. It goes without saying that perfusion of the skin envelope must tolerate this. In patients with severe hypertrophy and greater ptosis, we would recommend a two-stage approach with expander reconstruction, as is also described in the literature [17].

\section{Conclusions}

With proper patient selection, good aesthetic results can be achieved with both the inframammary and the radial approach. In the case of ptosis, we primarily recom- mend the radial approach; if a larger implant is used, an inframammary approach is also possible. If there is any doubt, expander reconstruction should always be the method of choice, especially with the inframammary approach, as our data demonstrate.

\section{Statement of Ethics}

This study was approved by the local ethics committee.

\section{Disclosure Statement}

The authors have no conflicts of interest to disclose.

\section{References}

1 Nahabedian MY. Implant-based breast reconstruction: strategies to achieve optimal outcomes and minimize complications. J Surg Oncol. 2016 Jun;113(8):895-905.

2 Cordeiro PG, Jazayeri L. Two-Stage ImplantBased Breast Reconstruction: An Evolution of the Conceptual and Technical Approach over a Two-Decade Period. Plast Reconstr Surg. 2016 Jul;138(1):1-11.

3 Albornoz CR, Bach PB, Mehrara BJ, Disa JJ, Pusic AL, McCarthy CM, et al. A paradigm shift in U.S. breast reconstruction: increasing implant rates. Plast Reconstr Surg. 2013 Jan; 131(1):15-23.

4 Cordeiro PG, McCarthy CM. A single surgeon's 12-year experience with tissue expander/implant breast reconstruction: part I. A prospective analysis of early complications. Plast Reconstr Surg. 2006 Sep;118(4):825-31.

5 Fischer JP, Wes AM, Tuggle CT 3rd, Serletti JM, Wu LC. Risk analysis of early implant loss after immediate breast reconstruction: a review of 14,585 patients. J Am Coll Surg. 2013 Dec;217(6):983-90.

6 Pinsolle V, Grinfeder C, Mathoulin-Pelissier S, Faucher A. Complications analysis of 266 immediate breast reconstructions. J Plast Reconstr Aesthet Surg. 2006;59(10):1017-24.
7 Bertozzi N, Pesce M, Santi P, Raposio E. OneStage Immediate Breast Reconstruction: A Concise Review. Biomed Res Int. 2017;2017: 6486859.

8 Agusti A, Ward A, Montgomery C, Mohammed K, Gui GP. Aesthetic and oncologic outcomes after one-stage immediate breast reconstruction using a permanent biodimensional expandable implant. J Plast Reconstr Aesthet Surg. 2016 Feb;69(2):211-20.

9 Nahabedian MY. Breast reconstruction: a review and rationale for patient selection. Plast Reconstr Surg. 2009 Jul;124(1):55-62.

10 Chen CM, Disa JJ, Sacchini V, Pusic AL, Mehrara BJ, Garcia-Etienne CA, et al. Nipplesparing mastectomy and immediate tissue expander/implant breast reconstruction. Plast Reconstr Surg. 2009 Dec;124(6):1772-80.

11 Cordeiro PG, McCarthy CM. A single surgeon's 12-year experience with tissue expander/implant breast reconstruction: part II. An analysis of long-term complications, aesthetic outcomes, and patient satisfaction. Plast Reconstr Surg. 2006 Sep 15;118(4):832-9.

12 Casella D, Di Taranto G, Marcasciano M, Sordi S, Kothari A, Kovacs T, et al. Nipple-sparing bilateral prophylactic mastectomy and immediate reconstruction with TiLoop ${ }^{\circledR}$ Bra mesh in BRCA1/2 mutation carriers: a prospective study of long-term and patient reported outcomes using the BREAST-Q. Breast. 2018 Jun;39:8-13.
13 Ramon Y, Ullmann Y, Moscona R, Ofiram E, Tamir A, Har-Shai Y, et al. Aesthetic results and patient satisfaction with immediate breast reconstruction using tissue expansion: a follow-up study. Plast Reconstr Surg. 1997 Mar;99(3):686-91.

14 Cavalcante FP, Lima MV. Nipple-sparing mastectomy with periareolar incision and two-stage reconstruction: initial analysis of 31 cases. Breast J. 2018 Nov;24(6):940-3.

15 Gschwantler-Kaulich D, Leser C, Salama M, Singer CF. Direct-to-implant breast reconstruction: higher complication rate vs cosmetic benefits. Breast J. 2018 Nov;24(6):95764.

16 Didier F, Radice D, Gandini S, Bedolis R, Rotmensz N, Maldifassi A, et al. Does nipple preservation in mastectomy improve satisfaction with cosmetic results, psychological adjustment, body image and sexuality? Breast Cancer Res Treat. 2009 Dec;118(3):623-33.

17 Yalanis GC, Nag S, Georgek JR, Cooney CM, Manahan MA, Rosson GD, et al. Mastectomy weight and tissue expander volume predict necrosis and increased costs associated with breast reconstruction. Plast Reconstr Surg Glob Open. 2015 Aug;3(7):e450. 